\title{
DNA Vaccines: The New Directions to Allergic Diseases
}

\author{
Zhi-Qiang Liu ${ }^{1,2}$ and Peng-Yuan Zheng ${ }^{1^{*}}$ \\ ${ }^{1}$ Department of Gastroenterology, The Fifth Affiliated Hospital of Zhengzhou University, Zhengzhou, Henan 450052, China \\ 2Shenzhen Key Laboratory of Ear, Nose and Throat (ENT), Department of Rhinology, Institute of ENT, Longgang ENT Hospital, Shenzhen, Guangdong, China
}

"Corresponding author: Peng-Yuan Zheng, Department of Gastroenterology, The Fifth Affiliated Hospital of Zhengzhou University, Zhengzhou, Henan 450052, China, E-mail: medp7123@126.COM

Received date: April 19, 2016; Accepted date: April 23, 2016; Published date: April 27, 2016

Copyright: $\odot 2016$ Liu ZQ, et al. This is an open-access article distributed under the terms of the Creative Commons Attribution License, which permits unrestricted use, distribution, and reproduction in any medium, provided the original author and source are credited

\section{Editorial}

Allergy, also known as type I hypersensitivity, is the condition that immune system hypersensitive to some foreign proteins and antigens that are normally not deleterious in most people [1,2]. Allergic diseases including food allergy, asthma, allergic dermatitis and other atopic disorders have increased greatly in the past decades. More than $20 \%$ of population had allergic rhinitis, $6 \%$ of people suffered by food allergy, and $20 \%$ affected by atopic dermatitis [3]. The underlying mechanisms involve antigen presenting cells such as dendritic cells initiating a skewed Th2 cell proliferation in situ and latterly immunoglobulin E antibodies produce by B cells [4]. It still lacks of efficient treatments for allergies, available medications in the clinic include symptomatic treatments to block the action of allergic mediators and cells degranulation such as antihistamines, glucocorticoids and epinephrine etc., another more useful management to some types of allergies that is capable of altering of the natural course of allergic diseases is specific immunotherapy (SIT), i.e. subcutaneous and sublingual immunotherapy [5-7], it involves gradually increase the amounts of allergen exposure to induce symptomatic relief. The mechanism for SIT is still being elucidated, possibly including induction of Treg, Th1 and blocking IgG antibodies [7,8], allergen extracts used in SIT unavoidably contain large amounts of nonallergenic materials that have been shown to prime Th2 cells activity, sporadic IgE mediated side effects and sometimes systemic anaphylaxis as well [9].

With the development of biological and genetic engineering techniques, DNA vaccination has coming into ours horizon, and opened novel approaches for protective and therapeutic treatment of allergic diseases [10,11]. DNA vaccination was first introduced in 1992 by Tang et al. [12], after the injection of RNA and DNA expression vectors into mouse. DNA vaccination is a technique to protect against disease through injecting genetically engineered DNA and uptake by cells to produce an antigen directly, inducing a protective immunological response. There have been some promising preclinical researches in DNA vaccines for viral, bacterial infections, allergy and autoimmune diseases, and some types of malignant tumors, showing potential advantages over conventional vaccines [13], for example, DNA vaccine is inexpensive and ease of development and production, no risk for infection by using subunit vaccination, the encoding antigen can be presented by both MHC I and MHC II, polarizing Th1 or Th2 type responses and long term persistence of immunogen. The immunological principles for DNA vaccination also involve the exogenous antigens uptake and processing by scavenger cells such as dendritic cells (DC), initiating the following immune response: DNA vaccine is taken up by DC and then transcribed and translated into encoded protein, denatured and degraded by various proteases and presented by the peptide- MHC I or MHC II complexes, thus can be recognized by specific $\mathrm{T}$ cells [14]. Latterly, researches revealed that transfected DNA vaccine may be recognized by a cytosolic DNA sensor and activate the TBK1-STING signal, resulting in the type I interferons production.

The pathogenesis of allergic disorders associates with imbalance of Th1 and Th2 lymphocyte subsets and antigen specific regulatory $\mathrm{T}$ cells deficiency, leading to an increase in the Th2 cells bias. Th2 cells secreting IL-4, IL-5 and IL-13 promote B cells IgE production. DNA vaccine offers a promising new approach for improving the specific immunotherapy effect by altering the allergic immune response [15]. In contrast with clinical SIT with allergen extracts to foster blocking IgG antibodies that can mask allergic epitopes, the DNA vaccines can induce a Th1 type response [16]. Studies demonstrated that DNA vaccines encoding only $\mathrm{T}$ cell epitopes could suppress allergic reactions as effectively as those encoding whole allergens, while protein immunization mainly rendered a Th2 type response. Amount of studies have introduced new methods to enhance the DNA vaccines efficiency for their easily modification in the treatment of allergic disease in last decades [14,17]. From the vector selection, harmonization of the allergen genes codon usage to co-injection of CpG-ODNs as adjuvants or cytokines co-expression was developed. Another strategy to overcome possible anaphylactic responses and guarantee the recruitment of antigen specific Th1 cells response, DNA vaccines was constructed by encoding hypoallergenic allergen derivatives while $\mathrm{T}$ cell epitopes still remained. Recently, Tsujimura $\mathrm{Y}$ et al. have established a mycobacterium secreted antigen-Ag85B and an IL-4 antagonistic mutant DNA vaccine and evaluated their curative effect in asthma mouse model [18]. Another DNA vaccine was constructed by inserting Cry j 2 antigen from Japanese red cedar into the lysosomal associated membrane nucleic acid sequence (JRCLAMP-Vax), and underwent phase I clinical trial [19].

DNA vaccines still have some limitations and safety concerns, which are only limited to protein immunogens, may induce antibody against DNA and immunologic tolerance. Numerous clinical studies showed DNA vaccination has no serious adverse events, while before its clinical application, this prophylactic and curable vaccination must be tested in therapeutic trials. With better understanding of the mechanisms involved in the type I hypersensitivities and optimizing approaches in DNA vaccine construction, DNA vaccines are certain to evolve into available strategy for the treatment of allergic diseases in the future.

\section{References}

1. Weidinger S, Novak N (2016) Atopic dermatitis. Lancet 387: 1109-1122.

2. Valenta R, Hochwallner H, Linhart B, Pahr S (2015) Food allergies: the basics. Gastroenterology 148: 1120-1131. 
Citation: Liu ZQ, Zheng PY (2016) DNA Vaccines: The New Directions to Allergic Diseases. J Allergy Ther 7: e114. doi: 10.4172/2155-6121.1000e114

Page 2 of 2

3. Platts-Mills TA, Erwin E, Heymann P, Woodfolk J (2005) Is the hygiene hypothesis still a Viable explanation for the increased prevalence of asthma? Allergy 79:25-31.

4. Syed A, Kohli A, Nadeau KC (2013) Food allergy diagnosis and therapy: where are we now? Immunotherapy 5: 931-944.

5. Calderon MA, Kleine-Tebbe J, Linneberg A, De Blay F, Hernandez Fernandez de Rojas D, et al. (2015) House Dust Mite Respiratory Allergy: An Overview of Current Therapeutic Strategies. J Allergy Clin Immunol Pract 3: 843-855.

6. Wood RA (2016) Food allergen immunotherapy: Current status and prospects for the future. J Allergy Clin Immunol 137: 973-982.

7. Valenta R, Campana R, Focke-Tejkl M, Niederberger V (2016) Vaccine development for allergen-specific immunotherapy based on recombinant allergens and synthetic allergen peptides: Lessons from the past and novel mechanisms of action for the future. J Allergy Clin Immunol 137: 351-357.

8. Linhart B, Valenta R (2012) Vaccines for allergy. Current opinion in immunology 24: 354-360.

9. Focke-Tejkl M, Valenta R (2012) Safety of engineered allergen-specific immunotherapy vaccines. Curr Opin Allergy Clinical immunol 12: 555-563.

10. Lu S (2014) Novel vaccine technology and the future of vaccine science. Hum Vaccin Immunother 10: 1143-1146.
11. Khan KH (2013) DNA vaccines: roles against diseases. Germs 3: 26-35.

12. Tang DC, DeVit M, Johnston SA (1992) Genetic immunization is a simple method for eliciting an immune response. Nature 356:152-154.

13. Coban C, Kobiyama K, Jounai N, Tozuka M, Ishii KJ (2013) DNA vaccines: a simple DNA sensing matter? Hum Vaccin Immunother 9: 2216-2221.

14. Li L, Petrovsky N (2016) Molecular mechanisms for enhanced DNA vaccine immunogenicity. Expert Rev Vaccines 15: 313-329.

15. Isakovic A, Weiss R, Thalhamer J, Scheiblhofer S (2014) Protective and therapeutic DNA vaccination against allergic diseases. Methods Mol Biol 1143: 243-258

16. Weiss R, Hammerl P, Hartl A, Hochreiter R, Leitner WW, et al. (2005) Design of protective And therapeutic DNA vaccines for the treatment of allergic diseases. Curr Drug Targets Inflamm Allergy 4: 585-597.

17. Williams JA (2014) Improving DNA vaccine performance through vector design. Curr Gene therapy 14:170-189.

18. Tsujimura Y, Yasutomi Y (2016) Allergy Vaccines Using a Mycobacterium-Secreted Antigen,Ag85B, and an IL-4 Antagonist. Methods Mol Biol 1403: 723-738.

19. Weiss R, Scheiblhofer S, Thalhamer J (2015) What is the antiallergic potential of DNA vaccination? Immunotherapy 7: 587-590. 